\title{
Image Techniques for Identifying Sea-Ice Parameters
}

\author{
Qin Zhang Roger Skjetne
}

Department of Marine Technology, Norwegian University of Science and Technology, 7491 Trondheim, Norway. E-mails: \{from,Qin.Zhang, Roger.Skjetne\} @ntnu.no

\begin{abstract}
The estimation of ice forces are critical to Dynamic Positioning (DP) operations in Arctic waters. Ice conditions are important for the analysis of ice-structure interaction in an ice field. To monitor sea-ice conditions, cameras are used as field observation sensors on mobile sensor platforms in Arctic. Various image processing techniques, such as Otsu thresholding, k-means clustering, distance transform, Gradient Vector Flow (GVF) Snake, mathematical morphology, are then applied to obtain ice concentration, ice types, and floe size distribution from sea-ice images to ensure safe operations of structures in ice covered regions. Those techniques yield acceptable results, and their effectiveness are demonstrated in case studies.
\end{abstract}

Keywords: Image processing; Sea-ice; ice concentration Floe size distribution; Aerial sea-ice image; Fisheye sea-ice image; 360 degree camera system.

\section{Introduction}

In 2008, the U.S. Geological Survey (USGS) estimated that the area north of the Arctic circle has 90 billion barrels of undiscovered, technically recoverable oil, 1,670 trillion cubic feet of technically recoverable natural gas, and 44 billion barrels of technically recoverable natural gas liquids in 25 geologically defined potential areas. These resources account for about 22 percent of the undiscovered, technically recoverable resources in the world. The Arctic accounts for about 13 percent of the undiscovered oil, 30 percent of the undiscovered natural gas, and 20 percent of the undiscovered natural gas liquids in the world. About 84 percent of the estimated resources are expected to occur offshore (Bird, 2008).

The exploration of the Arctic for petroleum is more technically and physically challenging than for any other environment. However, with improved technology and continuing high oil prices, the region is now receiving interest of the petroleum industry. The Norwegian supplier industry is thus seeing an increased de- mand for vessels capable of performing Dynamic Positioning (DP) operations in Arctic ice-covered areas. It is expected that such operations will be more frequent in the future due to exploration drilling, the possibility for hydrocarbon production, and other emerging advanced marine operations north of the polar circle. However, despite a few expeditions involving DP operations in ice conditions, in practice there are today no experience and little knowledge on DP operations in Arctic ice conditions available, neither in public domain nor among companies. Therefore, the development of new knowledge and insights into technological methods, operational procedures, and limitations in the Arctic environment are essential for performing safe commercial operations in a very harsh and ecologically sensitive Arctic environment. Many research works on Arctic technology and operations have been done, such as Gürtner (2009); Keinonen et al. (2000); Nguyen et al. (2009).

In the Arctic region, automatic DP operations are challenging since the behavior of ice forces is significantly different from the other environmental forces 


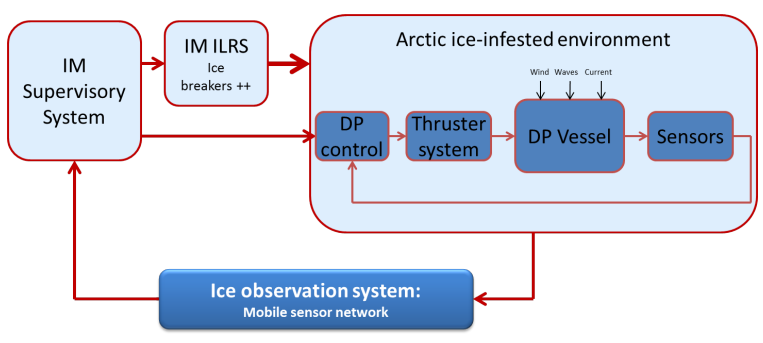

Figure 1: KMB Arctic DP system topology proposal.

that the DP systems are designed to compensate. Figure 1 gives the topology proposal of Arctic DP systems. The Arctic DP vessels in wide range of ice conditions usually depend on ice management that ice breakers break the incoming ice into smaller pieces to reduce the ice loads on the ship hull. By maintaining the heading towards the direction of ice drift motion, the operating vessel is able to minimize the loads on the hull, by only letting the smallest part of the vessel to undergo friction from the broken ice. The control model of the managed ice is designed to simulate the ice-vessel interaction. Then the controller is constructed for the vessel to cope with the large variations in managed ice loads, and maintain its position and heading (Makrygiannis, 2012).

Ice concentration, ice floe size distribution, ice types are important parameters in the field observations of sea-ice parameters to estimate ice loads. The development of temporally and spatially continuous field observations of sea-ice conditions is very important to mitigate the risk of such operations. One of the best ways to observe ice conditions in the oceans is by using imagery and applying digital image processing techniques to the observations. This method can reduce or suppress ambiguities, incompleteness, uncertainties, and errors regarding an object and its environment, yielding more accurate and reliable information (Haugen et al., 2011). Digital visual image techniques have been applied to ice observation. In Ji et al. (2011), a sea-ice digital image collection and processing system was utilized to monitor the ice parameters in the JZ20-2 oil-gas field of the Liaodong Bay.

Therefore, the use of cameras as sensors on mobile sensor platforms (e.g. unmanned vehicles) in Arctic DP operations will be explored for ice motion monitoring to characterize ice conditions as shown in Figure 2 (Zhang et al., 2012a). It has the potential of continuous measurements with high precision, which is particularly important for providing detailed localized information of sea-ice to ensure safe operations of structures in ice covered regions.

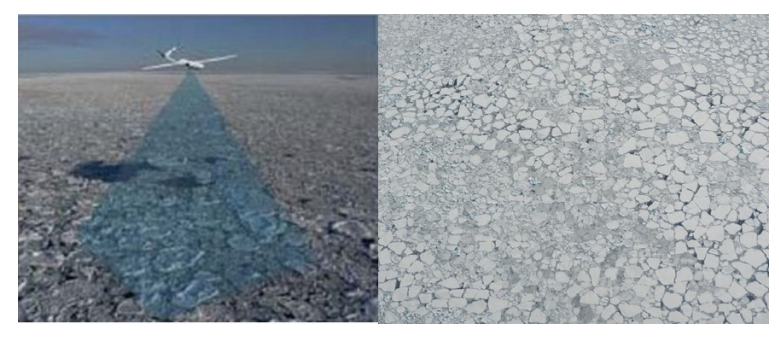

Figure 2: Image processing system in support to Arctic DP operation. The cameras are used as sensors on the Unmanned Aerial Vehicle (UAV) to monitor the conditions of sea-ice.

\section{Image processing methods}

\subsection{Ice pixel detection}

Usually, ice pixels have similar intensity, and have higher intensity values than those belonging to water in a uniformly illuminated ice image. The thresholding method turns a grayscale image into a binary image, e.g. in our case finds an appropriate grayscale theshold value which separates an ice image into an "ice region" and a "water region". The Otsu thresholding (Otsu, 1975), which is used to perform histogram shape-based image thresholding automatically, is one of the most common threshold segmentation algorithms. This algorithm assumes that the histogram (the distribution of gray-values) is bimodal and that the illumination is uniform. It then divides the histogram into two classes (i.e., the pixels are identified as either foreground or background) and finds the threshold value that minimizes the within-class variance.

Another ice pixel detection method is k-means clustering, which is a statistical data analysis technique that minimizes the within-cluster sum of distance to partition a set of data into groups (MacQueen et al., 1967). By using this method, the image is divided into three or more clusters. The cluster with the lowest average intensity value is considered to be water, while the other clusters are considered ice (Zhang et al., 2012a; Zhang and Skjetne, 2014). The k-means method is a good way for a quick review of data, especially if the objects are classified into many clusters (Basak et al., 1988).

The ice pixel detection using the Otsu thresholding method is similar to the detection using the k-means method by dividing the image into two clusters when the intensity values of all the ice pixels are significantly higher than water pixels (Zhang et al., 2012b). However, the Otsu thresholding method can only find "light ice" pixels. The "dark ice" (e.g. ice that are submerged in water), whose pixel intensity values are close to water pixels, may be lost. The k-means clustering 
method, on the other hand, has a better detection by dividing the image into three or more clusters, as shown in Figure 3.

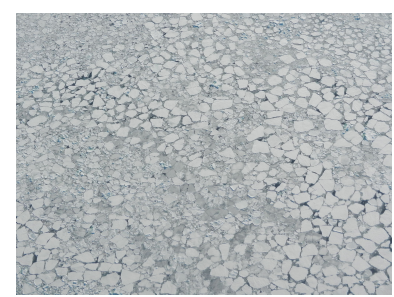

(a) Original sea-ice image.

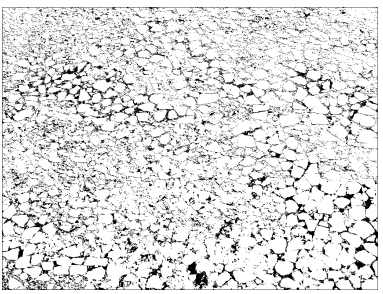
means method with 3 clusters. (c) Ice detection using $\mathrm{k}$ -

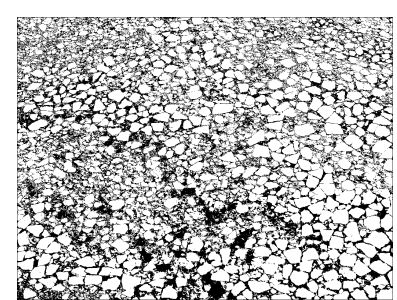

(b) "Light ice", detected by the Otsu thresholding method.

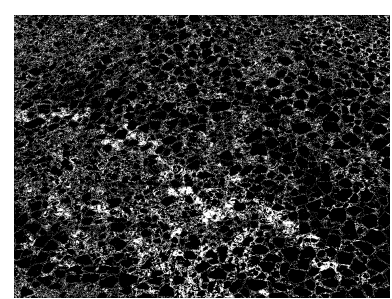

(d) "Dark ice", the difference between Figure 3(b) and 3(c).
Figure 3: Ice pixel detection.

\subsection{Floe boundary detection}

The floe size distribution is a basic parameter of sea-ice that affects the behavior of sea-ice extent both dynamically and thermodynamically (Toyota and Enomoto, 2002). It is also important in ice management for Arctic offshore operations (Keinonen, 2008; Hamilton et al., 2011). Automatic identification of individual floe boundaries is a key tool for extracting information of floe size distribution from sea-ice images. In an actual ice-covered environment, ice floes typically touch each other, and the junctions may be difficult to identify in digital images. This issue challenges the boundary detection of individual ice floes, and it significantly affects ice floe size analysis. Several methods, such as derivative boundary detection (Zhang et al., 2012b,a), morphology-based method (Zhang et al., 2012b,a; Banfield, 1991; Banfield and Raftery, 1992), watershedbased algorithms (Blunt et al., 2012; Zhang et al., 2013), have been applied to identify the floe boundaries. However, those methods are limited when trying to identify a boundary that is hidden. Zhang et al. (2014) and Zhang and Skjetne (2014) introduce the Gradient Vector Flow (GVF) Snake algorithm (Xu and Prince, 1998), which has a good detection capability of weak boundaries. Together with the distance transform (Rosenfeld and Pfaltz, 1968), this is used to iden-

tify ice floes and their boundaries automatically.

To evolve the GVF Snake correctly for ice floe boundary detection, a proper initial contour is required. Zhang and Skjetne (2014) showed that the initial contour close to the actual floe boundary, located inside the floe and centered as close to the ice floe center is most effective. To accomplish the requirements of the initial contour without manual interaction, an automatic contour initialization algorithm based on the distance transform and its local maxima is proposed. This is concluded as:

Step 1: Convert the ice image into binary image after separating the ice from the water (Zhang et al., 2014; Zhang and Skjetne, 2014), in which case the pixels with value ' 1 ' indicate ice, and pixels with value ' 0 ' indicates water; see Figure 4(a).

Step 2: Perform the distance transform to the binary image. Find the local maxima shown as the green numerals in Figure 4(b).

Step 3: Merge those local maxima within a short distance by using a dilation operator (Gonzalez et al., 2003). Find the 'seeds' that are centers of the dilated regions, shown as red ' + ' in Figure 4(b) and Figure $5(\mathrm{~b})$.

Step 4: Initialize the contours to be located at the seeds with the circular shape. The radius of the circle is then chosen according to the pixel value at the seed in the distance map; see the blue circles in Figure 4(b) Figure 5(b).

\begin{tabular}{|l|l|l|l|l|l|l|l|}
\hline 0 & 0 & 0 & 0 & 0 & 0 & 0 & 0 \\
\hline 0 & 1 & 1 & 1 & 1 & 1 & 0 & 0 \\
\hline 0 & 1 & 1 & 1 & 1 & 1 & 1 & 0 \\
\hline 0 & 1 & 1 & 1 & 1 & 1 & 1 & 0 \\
\hline 0 & 0 & 1 & 1 & 1 & 1 & 1 & 0 \\
\hline 0 & 0 & 1 & 1 & 1 & 1 & 0 & 0 \\
\hline 0 & 0 & 0 & 0 & 1 & 1 & 0 & 0 \\
\hline 0 & 0 & 0 & 0 & 0 & 0 & 0 & 0 \\
\hline
\end{tabular}

(a) Binary image matrix.

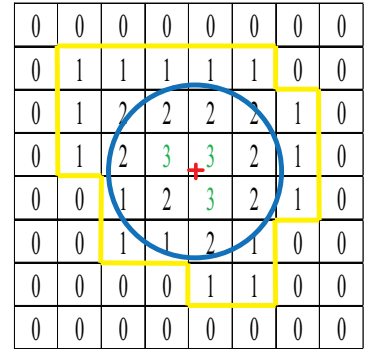

(b) Distance transform of Figure 4(a), local maximum, seed, and initial contour.

Figure 4: Contour initialization algorithm.

The circular shape is chosen as the shape of the initial contour, since this shape deforms to the floe boundary more uniformly than other shapes, being unaware of the floes irregular shape and orientation. This method ensure that the initial contours are located inside the floe and adapted to the floe size.

After initializing the contours, the GVF Snake algorithm is run on each contour to identify the floe boundary. Superimposing all the boundaries over the binarized ice image results in separation of the connected 


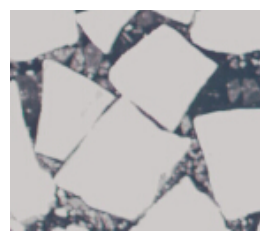

(a) Sea-ice floe image.

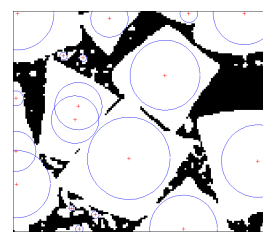

im- (c) Segmentation reage with initial sult, individual floes contours.

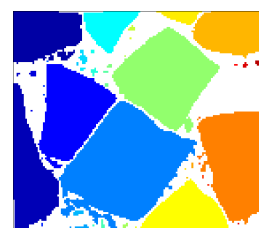
are labeled in different colors.
Figure 5: Connected ice floe segmentation based on GVF Snake.

ice floes, as shown in Figure 5.

\subsection{Floe shape enhancement}

After boundary detection, some segmented floes may contain holes or smaller ice floes inside. This means that the ice floe cannot be completely identified, and the shape of the segmented ice floe is rough. To smoothen the shape of the ice floe, morphological cleaning (Soh et al., 1998) is used after ice floe identification:

Step 1: Arrange all the segmented ice floes from small to large.

Step 2: Perform the morphological cleaning to the arranged ice floes in sequence.

This process will ensure the completeness of the ice floe and that smaller ice floes contained in larger floes are removed. The shape enhancement result is shown in Figure 6(c).

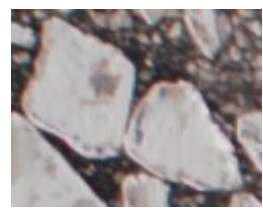

(a) Ice floe image (b) with speckle.

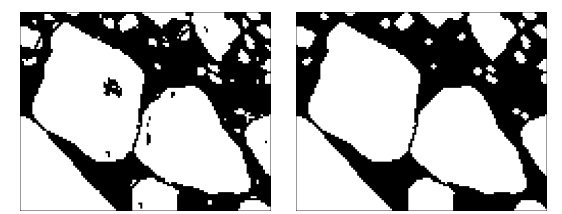

b) Segmentation (c) Shape enhanceresult of Figure ment result of $6(\mathrm{~b})$. $6(\mathrm{a})$.

Figure 6: Ice shape enhancement.

\section{Case Study}

\subsection{Case 1: Aerial sea-ice image processing}

A remote sensing mission to determine ice conditions was performed by the Northern Research Institute
(NORUT) at $78^{\circ} 55^{\prime} \mathrm{N} 11^{\circ} 56^{\prime} \mathrm{E}$, Hamnerabben, NyÅlesund from May 6th to 8th, 2011. An Unmanned Aerial Vehicle (UAV) was used as a mobile sensor platform, and a Canon EOS 450D camera was explored as a sensor on the UAV to measure ice statistics and properties. The UAV flew in the inner part of Kongsfjorden, and high resolution images of the sea-ice were collected.

Figure 7 shows an aerial image of the marginal ice zone. Since part of the ice pixels have intensity values close to water, both the "light ice" image and the "dark ice" image are used to obtain an accurate result. The procedure is:

Step 1: Derive the "light ice" image and "dark ice" image from Figure 7 by the method mentioned in Section 2.1

Step 2: Initialize the contours in both "light ice" image and "dark ice" image by the method mentioned in Section 2.2.

Step 3: Run the GVF Snake algorithm on the initial contours to derive "light ice" segmentation and "dark ice" segmentation individually.

Step 4: Collect all the ice pieces in both "light ice" and "dark ice" segmentations and perform the shape enhancement algorithm mentioned in Section 2.3.

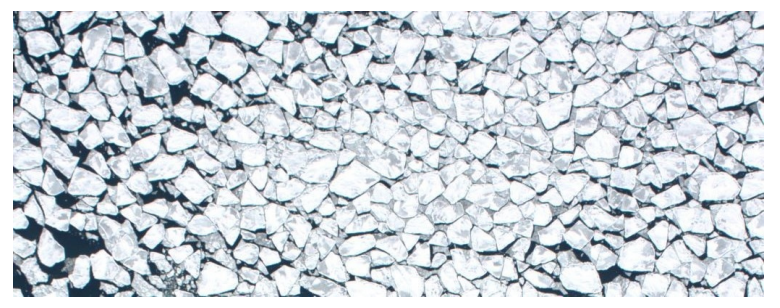

Figure 7: Aerial image of the marginal ice zone.

The result is four layers of a sea-ice image: ice floe (Figure 8(a)), brash ice (Figure 8(b)), slush (Figure $8(\mathrm{c})$ ), and water (Figure 8(d)). Based on the four layers, a total of 498 ice floes and 201 brash ice pieces are identified from Figure 7 . The coverage percentages are $76.73 \%$ ice floe, $0.46 \%$ brash ice, $9.05 \%$ slush, and $13.17 \%$ water.

The ice floe (brash) size can be determined by the number of pixels in the identified floe (brash). The ice floe and brash ice are labeled in different colors based on their sizes with ' $*$ '('.') at their centers, as shown in Figure 9. The resulting ice floe size distribution histogram is then shown in Figure 10.

\subsection{Case 2: Processing of shipboard sea-ice image with fisheye distortion}

The performance of KV Svalbard (a Norwegian Coast Guard icebreaker that operates in the Norwegian Arctic) in ice was studied by SAMCoT researchers, and a 


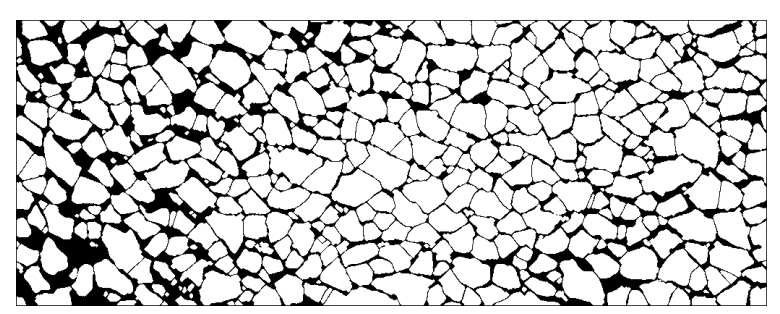

(a) Layer showing the "ice floes" of Figure 7

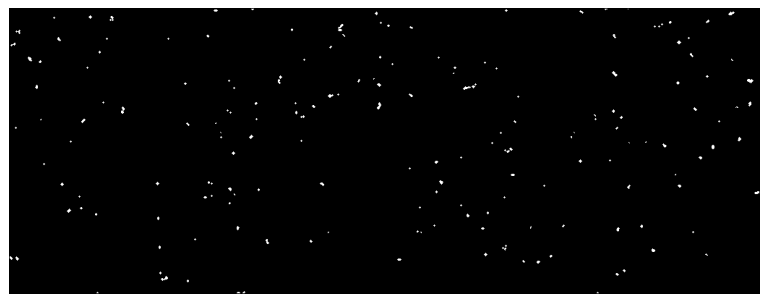

(b) Layer showing the "brash ice" of Figure 7

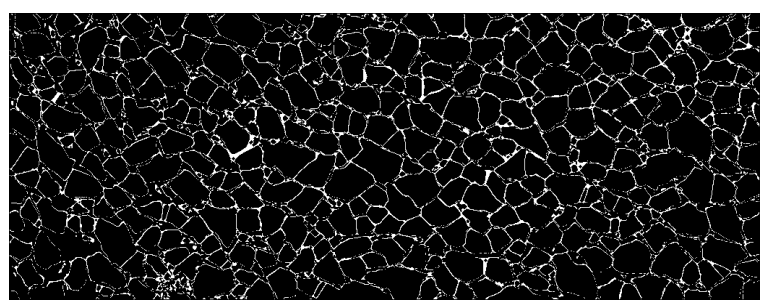

(c) Layer showing the "slush ice" of Figure 7

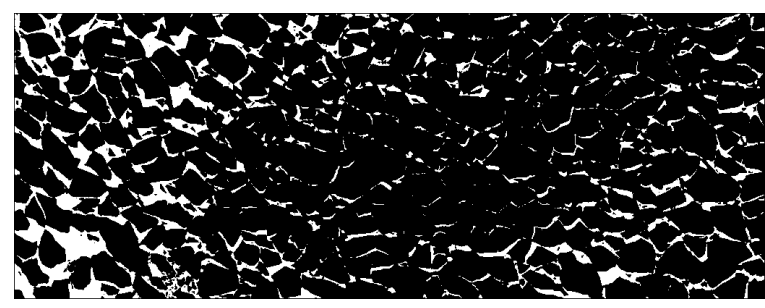

(d) Layer showing the "water" of Figure 7.

Figure 8: Identification result producing four layers for Figure 7.

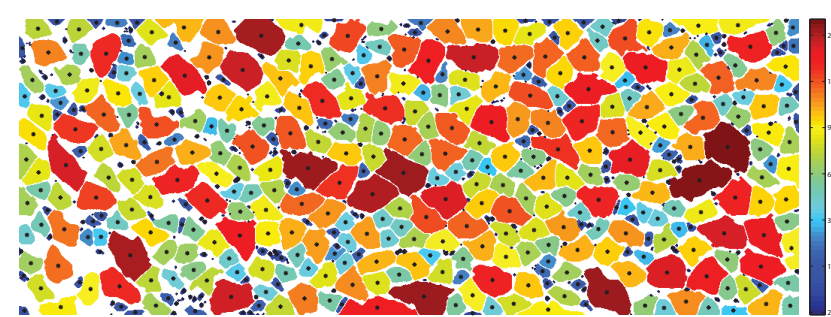

Figure 9: Floe and brash ice size distribution for Figure 7 .

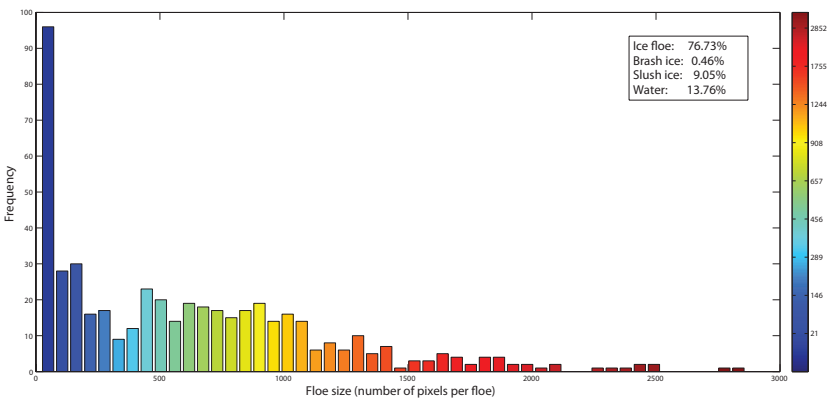

Figure 10: Floe size distribution histogram obtained from Figure 9.

set of full-scale experimental data was collected in the Fram Strait from the 8th to the 18th of March, 2012. In this cruise, GoPro HD HERO2 cameras were employed and mounted out of the hatch to capture the ice condition in front of KV Svalbard's bow during the experiments. Figure 11(a) shows a sample image of the downward looking camera (the perspective distortion can be ignored), and Figure 11(b) is its corresponding segmentation result of both "light" and "dark" ice by using Step 1 - 3 in Section 3.1.

The camera provided $170^{\circ}$ Field of View (FOV) angle (GoPro, accessed 2014-11-22) to give a wide view of the sea-ice at the expense of obtaining distorted images. The apparent effect of ice floes in the image is mapped around a sphere. The image magnification decreases with the distance from the optical axis. Besides the deformation, the size of the floe located in the center of the image is larger than it is closed to the image border. This affects the further ice floe size distribution results. To remove the fisheye distortion, the FOV angle is required by the method as illustrated in the Appendix. The "wide" FOV angle was measured approximately to $119^{\circ}$ (see Figure 17) by image width as apposed to the claimed $170^{\circ}$. The calibration should be performed after the detection of the floe boundaries (Section 2.2 and 3.1), since the calibrated image becomes enlarged and part of the image may be blurred. This may induce errors in the detection of the floe boundaries. After that, we perform the floe shape enhancement (Section 2.3) to the calibrated image to obtain the final floe size distribution result, as shown in Figure 12. All floes in Figure 12 are actually found from Figure 11(a), although some large floes have odd shapes. This is because their distorted shapes in Figure 11(a) are corrected by the fisheye calibration algorithm. About 29 ice floes and 9 brash ice pieces are found, and the ice concentration is calculated as $74.17 \%$. 


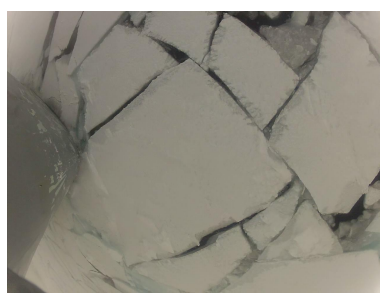

(a) Shipboard sea-ice image (b) Segmentation result of (a), with fisheye distortion. the white pixels are "light ice" pixels, and the grey pixels are "dark ice pixels.

Figure 11: Shipboard sea-ice image with fisheye distortion and its segmentation result.

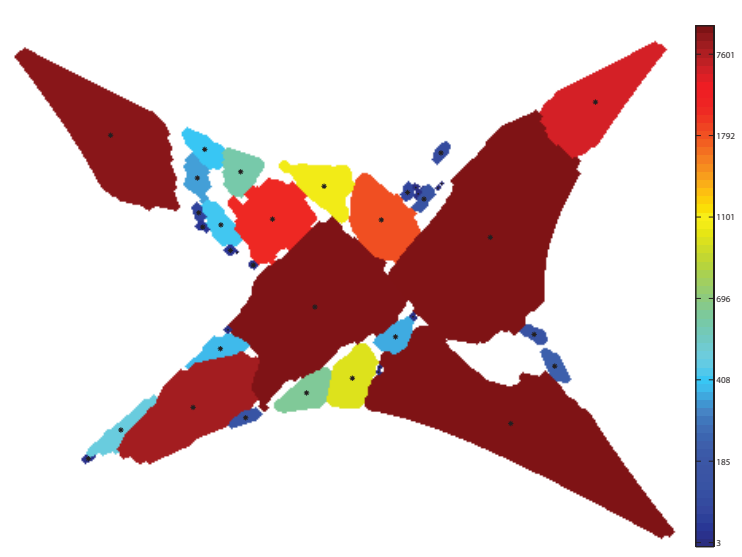

Figure 12: Floe and brash ice size distribution of Figure $11(\mathrm{~b})$.

\subsection{Case 3: The 360 degree camera system and ice image processing}

A 360 degree camera system was used in the Oden Arctic Technology Research Cruise (OATRC 2013) to monitor the ice condition around the vessel. This system consisted of two $360^{\circ}$ video camera units Arecont Vision 20365DN-HB, a 20 megapixel camera unit that includes a set of 4 independent cameras ( 4 objectives) with heater and blower housing option to provide normal camera functioning in cold and wet conditions. The system was installed on the helicopter deck of icebreaker Oden, allowing the construction of $360^{\circ}$ panorama images of ice conditions around the vessel (Bjørklund et al., 2013).

In order to stitch the individual camera images into a single panorama, Bjørklund et al. (2013) used the commercial software PTGui Pro v9.1.8. A set of pictures with clearly visible ice structures was selected and stitched manually to create the template. Based on this template, batches of images may then be stitched together automatically. To estimate the ice concen- tration around the icebreaker based on the panorama images, rectilinear (flat) projection that projects the image on to a plane is chosen to remove the geometric distortion. A resulting panorama image during the pushing test experiment is presented in Figure 13. Before applying the algorithms to these panorama images, one image per minute was found sufficient, and each image was fed to the program for further processing.

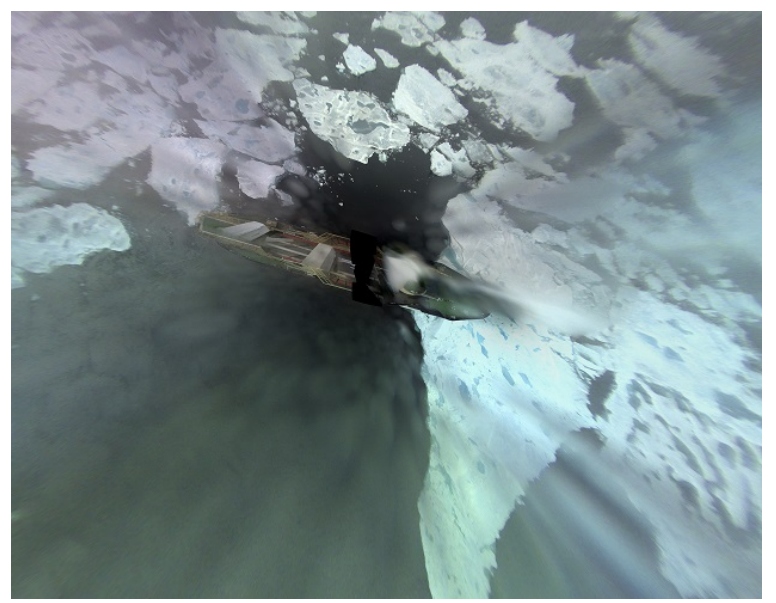

Figure 13: 360 degree panorama image.

Significant distortion exists in the resulting panorama images as a result of camera vibration and influence of the pitch and roll of the icebreaker during maneuvers. The images are stitched from a single template created from a particularly clear set of images. The resulting images are also highly blurred because of the occasional icing/fogging on the camera dome due to insufficient heating, as shown in Figure 13. The ice pixel detection by Otsu thresholding method is insufficient since the occasional icing/fogging parts are considered as ice pixels because of their high intensity. This will increase the manual work for the local processing. Instead, the $\mathrm{k}$-means method is used and divides the image into three clusters. Only the cluster with the highest average intensity value is considered to be ice, and the other clusters are considered water. Then, manual local processing are made by using the methods in Section 2.1 and 2.2 to identify all the missing ice and floe boundaries, resulting in a segmented image. After performing the shape enhancement method (Section 2.3) to the segmented image, 128 ice floes and brash ice pieces are founded. The ice concentration is calculated to 41.79\%, as presented in Figure 14.

By processing the sequence of panorama images obtained from the pushing test experiment, the ice concentration was calculated as a function of time and presented in Figure 15. 


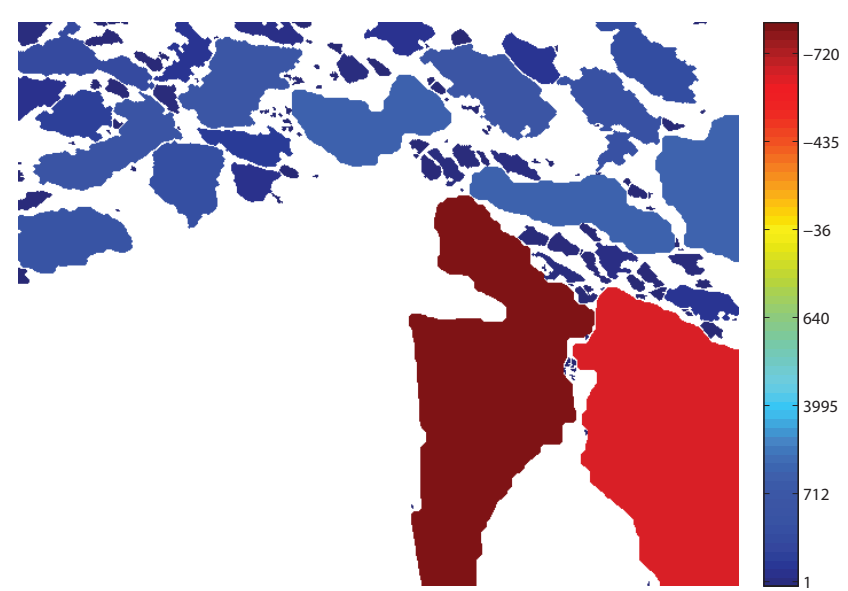

Figure 14: Identified ice floe distribution of Figure 13.

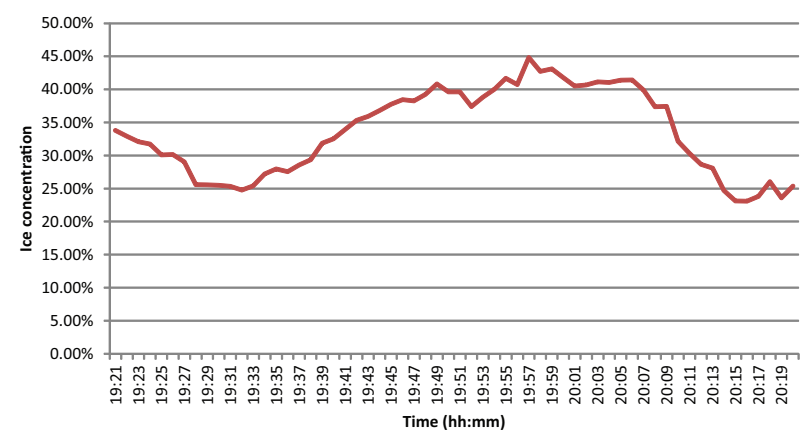

Figure 15: Time-varying ice concentration.

\section{Conclusion}

Various image techniques have been introduced and applied to the collected sea-ice images for analysis to give some results applicable to ice engineering and ice management. These techniques perform well in automatic processing of marginal ice zone images, while manual intervention may be necessary for processing other seaice images. More results and better information of sea ice from visual images will be investigated by further development of these image processing techniques.

\section{Acknowledgments}

Research partly funded by Research Council of Norway (RCN) KMB project no. 199567: "Arctic DP" with partners Kongsberg Maritime, Statoil, and DNV GL, and partly by RCN project no. 203471: CRI SAMCoT.

We appreciate the constructive comments to the paper from Petter Risholm (SINTEF ICT) and Øivin Aarnes (DNV GL) during review of the text.

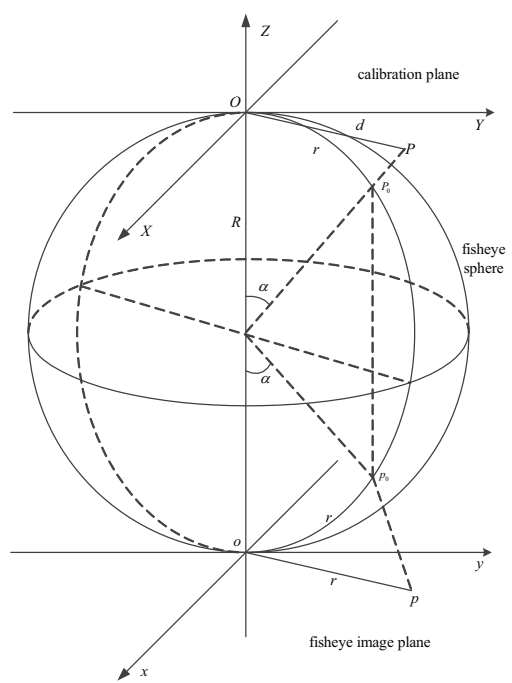

(a) 3-D spherical perspectiion

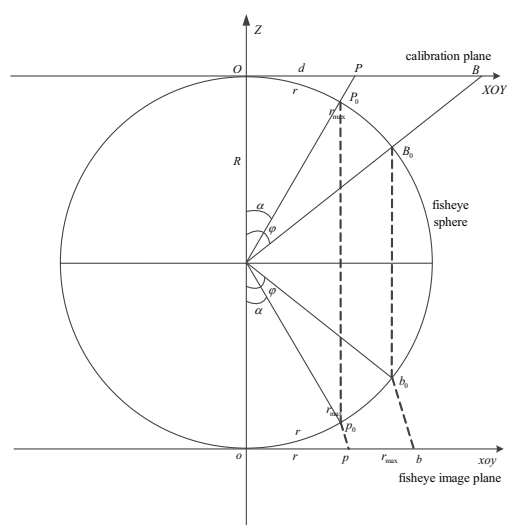

(b) 2-D spherical perspectiion

Figure 16: Fisheye calibration.

\section{Appendix: Fisheye calibration}

The location of any object in the image is a function of the Field of View (FOV) angle of the camera in relation to its calibrated flat surface. Figure 16 illustrates the mapping between fisheye image coordinates and the corresponding calibration coordinates.

The fisheye image coordinates, denoted with small letters $(x, y)$, is located at the bottom of the fisheye sphere and is perpendicular to the $Z$-axis (the optical axis of the lens). The calibration coordinates denoted with capital letters $(X, Y)$, are on the top of the fisheye sphere parallel to the image coordinates. The center of the fisheye image ' $O$ ' located in the $Z$-axis, acts as the origin of the image coordinates. The intersection point $O$ of the calibration plane and $Z$-axis, acts as the origin of the calibration coordinates. $\varphi$ is half the lens Field of View (FOV) angle, which is known. For any point $p(x, y)$ on the image plane and its corresponding point 
$P(X, Y)$ on the calibration plane, the radial distance $|\overrightarrow{O P}|$ in the distorted fisheye image plane $|\overrightarrow{o p}|$ is equivalent to the length of the arc segment $r$ between the $Z$-axis and $P_{0}$, which is the intersection point of the projection ray of the point $P$ and the fisheye sphere. Therefore,

$$
\begin{aligned}
|\overrightarrow{o p}| & =r=R \cdot \alpha \\
& =R \cdot \arctan \frac{|\overrightarrow{O P}|}{R},
\end{aligned}
$$

where $R$ is the radius of the fisheye sphere, obtained by

$$
R=\frac{r_{\max }}{\varphi}=\frac{r_{m} \cdot \mu}{\varphi},
$$

and $r_{\text {max }}$ is half the capture range of the fisheye image in length, $r_{m}$ is the pixel number in maximum semidiameter, obtained by counting half the number of pixels in the image length, and $\mu$ is the pixel magnification of the image. From Figure 16(a), we also find that

$$
\begin{aligned}
|\overrightarrow{o p}| & =\sqrt{x^{2}+y^{2}} \cdot \mu \\
|\overrightarrow{O P}| & =\sqrt{X^{2}+Y^{2}} \cdot \mu \\
\frac{x}{y} & =\frac{X}{Y}
\end{aligned}
$$

Substituting equations (2) - (5) into (1), the location of any point $P(X, Y)$ in the calibration coordinates can be determined from its fisheye image coordinates $p(x, y)$ by

$$
\begin{aligned}
& X=\frac{x}{\sqrt{x^{2}+y^{2}}} \cdot \frac{r_{m} \tan \frac{\varphi \sqrt{x^{2}+y^{2}}}{r_{m}}}{\varphi}, \\
& Y=\frac{y}{\sqrt{x^{2}+y^{2}}} \cdot \frac{r_{m} \tan \frac{\varphi \sqrt{x^{2}+y^{2}}}{r_{m}}}{\varphi} .
\end{aligned}
$$

From (6) we find that the relationship between the fisheye image coordinates and its calibration coordinates only depends on the camera's FOV angle $2 \varphi$. The magnification $\mu$ will not affect the relationship since it is counteracted based on equations (2) - (4). The calibration result is illustrated in Figure 17.

\section{References}

Banfield, J. Automated tracking of ice floes: A stochastic approach. Geoscience and Remote Sensing, IEEE Transactions on, 1991. 29(6):905-911. doi:10.1109/36.101369.

Banfield, J. D. and Raftery, A. E. Ice floe identification in satellite images using mathematical morphology
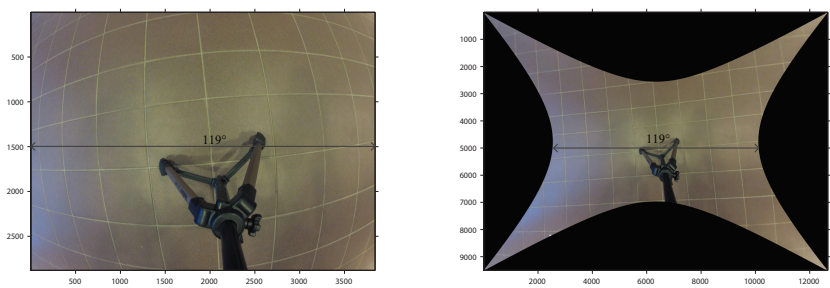

Figure 17: Fisheye calibration result

and clustering about principal curves. Journal of the American Statistical Association, 1992. 87(417):716. doi:10.1080/01621459.1992.10475169.

Basak, S. C., Magnuson, V., Niemi, G., and Regal, R. Determining structural similarity of chemicals using graph-theoretic indices. Discrete Applied Mathematics, 1988. 19(1):17-44. doi:10.1016/0166218X(88)90004-2.

Bird, K. J. Circum-Arctic resource appraisal: Estimates of undiscovered oil and gas north of the Arctic Circle. US Department of the Interior, US Geological Survey, 2008.

Bjørklund, H., Prusakov, A., and Sinitsyn, A. 360 degree camera system. Technical report, Department of Civil and Transport Engineering, Norwegian University of Science and Technology, 2013.

Blunt, J., Garas, V., Matskevitch, D., Hamilton, J., and Kumaran, K. Image analysis techniques for high Arctic, deepwater operation support. In OTC Arctic Technology Conference. Houston, Texas, USA, 2012.

Gonzalez, R. C., Woods, R. E., and Eddins, S. L. Digital Image Processing Using MATLAB. PrenticeHall, Inc., Upper Saddle River, NJ, USA, 2003. doi:10.1115/OMAE2012-83860.

GoPro. HD HERO2 PROFESSIONAL. accessed 2014-11-22. http://gopro.com/ product-comparison-hd-hero2-hd-hero-cameras.

Gürtner, A. Experimental and numerical investigations of ice-structure interaction. Ph.D. thesis, Norwegian University of Science and Technology, 2009.

Hamilton, J., Holub, C., Blunt, J., Mitchell, D., Kokkinis, T., et al. Ice management for support of arctic floating operations. In OTC Arctic Technology Conference. Offshore Technology Conference, 2011.

Haugen, J., Imsland, L., Løset, S., and Skjetne, R. Ice observer system for ice management operations. In Proceeding of the 21st International Ocean and Polar Engineering Conference. Maui, Hawaii, USA, 2011. 
Ji, S., Li, H., Wang, A., and Yue, Q. Digital image techniques of sea ice field observation in the bohai sea. In POAC11-077, Proceedings of the 21st International Conference on Port and Ocean Engineering under Arctic Conditions (POAC11), Montréal, Canada. 2011.

Keinonen, A. Ice management for ice offshore operations. In Proceedings of the Offshore Technology Conference. Houston, TX. 2008.

Keinonen, A., Wells, H., Dunderdale, P., Pilkington, R., Miller, G., Brovin, A., et al. Dynamic positioning operation in ice offshore sakhalin may-june 1999. In The Tenth International Offshore and Polar Engineering Conference. International Society of Offshore and Polar Engineers, 2000.

MacQueen, J. et al. Some methods for classification and analysis of multivariate observations. In Proceedings of the fifth Berkeley symposium on mathematical statistics and probability, volume 1. California, USA, page 14, 1967.

Makrygiannis, A. Design and Simulation of an IceCapable DP System. Master's thesis, Norwegian University of Science and Technology, The address of the publisher, 2012.

Nguyen, D. T., Sørbø, A., and Soerensen, A. Modelling and control for dynamic positioned vessels in level ice. In Manoeuvring and Control of Marine Craft. pages 229-236, 2009. doi:10.3182/20090916-3-BR3001.00036 .

Otsu, N. A threshold selection method from gray-level histograms. Automatica, 1975. 11(285-296):23-27. doi:10.1109/TSMC.1979.4310076.

Rosenfeld, A. and Pfaltz, J. L. Distance functions on digital pictures. Pattern Recognition, 1968. 1(1):3361. doi:10.1016/0031-3203(68)90013-7.

Soh, L.-K., Tsatsoulis, C., and Holt, B. Identifying ice floes and computing ice floe distributions in SAR images. In Analysis of SAR Data of the Polar Oceans, pages 9-34. Springer, 1998.

Toyota, T. and Enomoto, H. Analysis of sea ice floes in the sea of okhotsk using ADEOS/AVNIR images. In Proceedings of the 16th IAHR International Symposium on Ice, Dunedin, New Zealand. pages 211-217, 2002.

$\mathrm{Xu}, \mathrm{C}$. and Prince, J. L. Snakes, shapes, and gradient vector flow. IEEE Transactions on Image Processing, 1998. 7(3):359-369. doi:10.1109/83.661186.
Zhang, Q. and Skjetne, R. Image processing for identification of sea-ice floes and the floe size distribution. Submit to IEEE Transactions on Geoscience and Remote Sensing, 2014.

Zhang, Q., Skjetne, R., Løset, S., and Marchenko, A. Digital image processing for sea ice observation in support to Arctic DP operation. In Proceedings of 31st International Conference on Ocean, Offshore and Arctic Engineering, OMAE2012-83860. ASME, Rio de Janeiro, Brazil, 2012a.

Zhang, Q., Skjetne, R., Metrikin, I., and Løset, S. Image processing for ice floe analyses in broken-ice model testing. Submit to Cold Region Science and Technology, 2014.

Zhang, Q., Skjetne, R., and Su, B. Automatic image segmentation for boundary detection of apparently connected sea-ice floes. In Proceedings of the 22nd International Conference on Port and Ocean Engineering under Arctic Conditions. Espoo, Finland, 2013.

Zhang, Q., van der Werff, S., Metrikin, I., Løset, S., and Skjetne, R. Image processing for the analysis of an evolving broken-ice field in model testing. In Proceedings of 31st International Conference on Ocean, Offshore and Arctic Engineering, OMAE2012-84117. ASME, Rio de Janeiro, Brazil, 2012b. doi:10.1115/OMAE2012-84117. 